\title{
Frequency-Domain Hot-Wire Measurements of Molten Nitrate Salt Thermal Conductivity
}

\author{
Andrew Z. Zhao, Matthew C. Wingert, and Javier E. Garay* \\ Materials Science and Engineering Program \\ Mechanical and Aerospace Engineering Department \\ University of California, San Diego \\ La Jolla, CA 92093 \\ *Corresponding author: jegaray@ucsd.edu
}

\section{Supplemental Information}

\section{Appendix 1. Validity of neglecting radiation in the thermal model}

The model (Eqs. 2-4) we use to fit to measured data and extract sample thermal conductivity does not consider heat transfer by radiation. To confirm that radiation can be neglected in our thermal conductivity measurement, we calculate the relative significance of thermal radiation compared to thermal conduction between our sensor and molten salt during the measurement. Before the measurement, we let the temperature of our system equilibrate, so we assume the temperature of the sensor and the salt are equal. When we apply current to our hotwire, joule heating leads to a temperature difference between the wire and salt that will lead to radiative heat transfer.

To quantify the effect of radiative and conductive heat transport, we can weigh the relative heat fluxes from radiation, $Q_{\text {rad }}$ and conduction, $Q_{\text {cond }}$ defined as:

$$
\begin{gathered}
Q_{\text {rad }}=A_{\text {surface }} \sigma \varepsilon\left(T_{\text {wire }}^{4}-T_{a m b}^{4}\right) \approx 4 A_{\text {surface }} \sigma \varepsilon T_{a m b}^{3} \Delta T_{w} \\
Q_{\text {cond }}=\left.\kappa_{0} A_{\text {surface }} \frac{d \tilde{T}_{2 \omega}}{d r}\right|_{r 0}
\end{gathered}
$$

where the temperature rise of the wire is $\Delta T_{\text {wire }}=\widetilde{T}_{2 \omega, \text { avg }}$ as defined in this work and our previous paper $^{1}, \sigma$ is the Stefan-Boltzmann constant $\left(5.67 \mathrm{e}-8 \mathrm{~J} /\left(\mathrm{m}^{2} \mathrm{sK}^{4}\right)\right), A_{\text {surface }}$ is the surface area of the hot-wire, $r 0$ is the radius of the hot-wire, $T_{a m b}$ is the ambient background temperature, and $\varepsilon$ is the emissivity of the alumina surface of our hot-wire. We can assume blackbody radiation $(\varepsilon=1)$ 
to check the maximal radiative effect. The approximate ratio of heat fluxes due to radiation and conduction from the hot-wire is then:

$$
\frac{Q_{r a d}}{Q_{c o n d}} \approx \frac{4 \sigma T_{a m b}^{3}}{\kappa_{0}} \frac{\tilde{T}_{2 \omega, a v g}}{\left.\frac{d \tilde{T}_{2 \omega}}{d r}\right|_{r 0}}
$$

As mentioned earlier, we define the temperature rise, $\widetilde{T}_{2 \omega, \text { avg }}$ in the hot-wire using Eq.2. To calculate the conductive heat flux from the hot-wire into the molten salt, we take the radial gradient at the wire surface of the temperature rise as described in our previous work ${ }^{1}$ as follows:

$$
\begin{aligned}
& \tilde{T}_{2 \omega}(r)=\frac{1}{l} \int_{0}^{l} \widetilde{T}_{0}(r, z) d z=\sum_{n=1}^{\infty}\left[\frac{1}{l} \int_{0}^{l} K_{e}(n, z) d z\right]\left[A_{c, 0} I_{0}\left(\varphi_{0} r\right)+\sqrt{\frac{2}{l}}\left(\frac{\tilde{P}_{0}}{\pi r_{0}^{2} L \kappa_{0} \lambda_{n} \varphi_{0}^{2}}\right)\right] \\
& \left.\frac{d \tilde{T}_{2 \omega}}{d r}\right|_{r 0}=\sum_{n=1}^{\infty}\left(\frac{8 \tilde{P}_{R M S}}{\pi^{3} r_{0}^{2} L \kappa_{0} \varphi_{0}^{2}(2 n-1)^{2}}\right)\left(\frac{\kappa_{1} \varphi_{1}}{\kappa_{0}}\right)\left(\frac{\beta I_{1}\left(\varphi_{1} r_{0}\right)-\alpha K_{1}\left(\varphi_{1} r_{0}\right)}{\alpha \delta-\gamma \beta}\right)
\end{aligned}
$$

Finally, we calculate the ratio between radiation and conduction (Eq. A3), plotted in Figure S1.

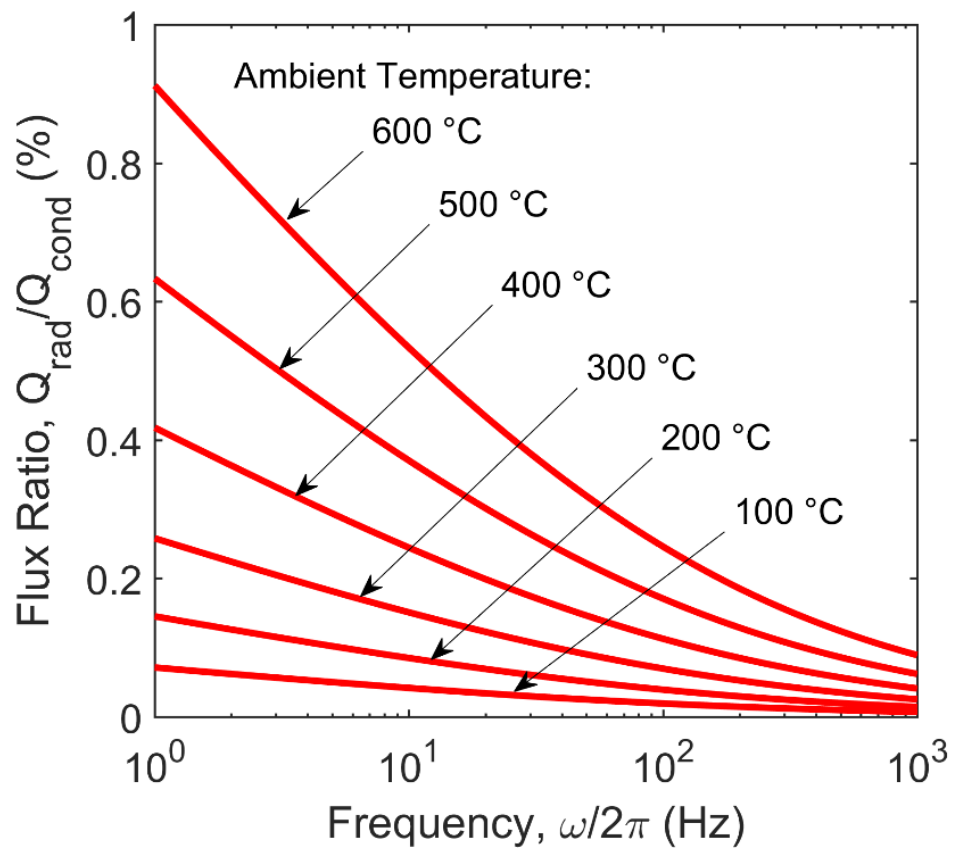

Figure S1. The percentage of radiation flux compared to conduction flux for various ambient temperatures as a function of input frequency. The radiation flux is less than $1 \%$ of the conduction flux and thus considered negligible. 
The highest temperature that our hot-wire system reached was $465^{\circ} \mathrm{C}(738.15 \mathrm{~K})$, shown in Figure 4, which is well below the $600^{\circ} \mathrm{C}(873.15 \mathrm{~K})$ upper limit shown in Fig. S1. We find that the $Q_{\text {rad }}$ is less than $1 \%$ of $Q_{\text {cond }}$ for the entire frequency range and for all salt temperatures below $600^{\circ} \mathrm{C}$ $(873.15 \mathrm{~K})$, validating our assumption that radiation does not play a significant role in our measurement of molten salt thermal conductivity

\section{Appendix 2. Checking the boundary condition at the wire ends}

Here we discuss the validity of the assumption of zero-temperature rise at the wire ends as a boundary condition that was used to develop Equation 2. For a full discussion on the model development please see our previous work. ${ }^{1}$ If the thermal conductance at the ends of the wire into the substrate is significantly larger than the thermal conductance into the sample or along the wire, then the heat dissipates into the high thermal conductivity substrate fast enough to keep a negligible temperature rise at the wire ends, validating the boundary condition. Thus, we examine the ratio of heat conductance from the Pt wire into the substrate, $G_{\text {substrate }}$ to the heat conduction into the sample, $G_{\text {sample }}$ as well as the heat conduction along the Pt wire, $G_{\text {wire }}$.

For our setup, the Pt wire is bonded/welded to the platinum leads on high thermal conductivity (alumina or sapphire) substrates. As conservative estimate, the heat conduction into the substrate, we assume conduction into the substrate is limited to the edge length of the welded portion of the wire, $L_{\text {weld }}$, and the thickness of the substrate, $d_{\text {substrate }}$. Therefore, for a molten salt thermal conductivity sample, $0.5 \mathrm{Wm}^{-1} \mathrm{~K}^{-1}$, the conductance ratio is $G_{\text {substrate }} / G_{\text {sample }} \approx$ $\left(2 \pi D L_{\text {weld }} \kappa_{\text {substrate }} / d_{\text {substrate }}\right) /\left(\pi D \kappa_{\text {sample }}\right)=\left(2 L_{\text {weld }} \kappa_{\text {substrate }}\right) /\left(\kappa_{\text {sample }} d_{\text {substrate }}\right)$.

Using the dimensions and properties, $L_{\text {weld }}=1 \mathrm{~mm}, d_{\text {substrate }}=1 \mathrm{~mm}, \kappa_{\text {substrate }}=30$ $W m^{-1} K^{-1}$ and $\kappa_{\text {substrate }}=0.5 \mathrm{Wm}^{-1} K^{-1}$ gives $G_{\text {substrate }} / G_{\text {sample }} \approx 120$.

Similarly, for the conductance ratio into the substrate versus along the Pt wire we have

$$
G_{\text {substrate }} / G_{\text {wire }} \approx\left(2 \pi D L_{\text {weld }} \kappa_{\text {substrate }} / d_{\text {substrate }}\right) /\left(\left(\frac{\pi}{2}\right) D^{2} \kappa_{\text {wire }} / L_{\text {weld }}\right)=
$$

$\left(4 L_{\text {weld }}^{2} \kappa_{\text {substrate }}\right) /\left(D \kappa_{\text {wire }} d_{\text {substrate }}\right)$. Using $D=0.025 \mathrm{~mm}$ and $\kappa_{\text {wire }}=60 \mathrm{Wm}^{-1} \mathrm{~K}^{-1}$, $G_{\text {substrate }} / G_{\text {wire }} \approx 80$. In both cases the estimated thermal conductance into the substrate is $\sim 100$ times larger than into the sample or past the substrate through the Pt wire. Thus, the boundary condition that the temperature rise at the wire ends is zero is valid for the conditions in this study 


\section{Appendix 3. Validity of assuming a uniform salt temperature}

To check if the temperature of the sample is uniform, we use a lumped capacitance model. ${ }^{2}$ The criterion for negligible temperature gradients within an object from the lumped capacitance model is that the Biot number (Bi) is less than 0.1, indicating that convective heat loss to the environment is very small compared to the conductive heat transfer within the sample.

The crucible containing the molten salt and sensor is a small volume of material and is suspended in the tube furnace. The sample is radiatively heated from the tube furnace walls with a primary cooling path of natural convection due to the Argon gas filling the tube furnace. For this analysis, we measure the temperature of the larger alumina tube that encases the sample holder in an argon environment, and we measure the temperature of the smaller alumina crucible that holds our molten salt sample and sensor (see Figure 3a).

Next, we use the highest temperature of our system and an energy balance to calculate the maximum convective heat transfer. During this work, the highest steady state temperature of the sample $\left(T_{\text {sample }}\right)$ was $468^{\circ} \mathrm{C}(742 \mathrm{~K})$ and the outer alumina tube temperature ( $\left.T_{\text {outer tube }}\right)$ was $500^{\circ} \mathrm{C}(773 \mathrm{~K})$. The energy balance of our sample, which includes the energy in, $Q_{\text {in }}$ from radiation from the larger alumina tube and the energy out, $Q_{\text {out }}$ from convective heat loss from the surface of the sample holder, can be written: $Q_{\text {in }}-Q_{\text {out }}=0$, where $Q_{\text {in }}=\sigma A \varepsilon_{1}\left(T_{\text {outer tube }}^{4}-\right.$ $\left.T_{\text {sample }}^{4}\right)$ and $Q_{\text {out }}=h A\left(T_{\text {sample }}-T_{\infty}\right)$. Thus,

$$
\sigma A \varepsilon_{1}\left(T_{\text {outer tube }}^{4}-T_{\text {sample }}^{4}\right)=h A\left(T_{\text {sample }}-T_{\infty}\right)
$$

where $\sigma$ is the Stefan-Boltzmann constant $\left(5.67 \times 10^{-8} \frac{J}{m^{2} s K^{4}}\right), A$ is the surface area of the sample holder (which cancels out), $\varepsilon_{1}$ is the emissivity of the alumina sample holder that we will conservatively assume to be $1, T_{\text {sample }}$ is the highest temperature that our molten salt reached $\left(T_{\text {sample }}=468^{\circ} \mathrm{C}=742 \mathrm{~K}\right.$ from Figure 4$), T_{\text {outer tube }}$ is the highest temperature we set for the 
outer tube $\left(T_{\text {outer tube }}=500^{\circ} \mathrm{C}=773 \mathrm{~K}\right)$, and $T_{\infty}$ is the ambient argon gas temperature $\left(T_{\infty}=\right.$ $\left.30^{\circ} \mathrm{C}=303 K\right)$

Note the gas is static and not flowing, and thus according to Eq. A5 we find the heat transfer coefficient for natural convection $h=6.96 \frac{\mathrm{W}}{\mathrm{m}^{2} K}$. Next, we find the Biot number using the definition:

$$
B i=\frac{h L_{c}}{\kappa}=\frac{h D}{4 \kappa}
$$

where $L_{c}=$ Volume/Surface Area $=D / 4$ is the characteristic length scale of the cylindrical sample holder ( $0.025 \mathrm{~m}$ diameter) and $\kappa$ is the thermal conductivity of the molten salt making up the majority of the volume of the sample holder $\left(\approx 0.5 \mathrm{Wm}^{-1} \mathrm{~K}^{-1}\right)$. Eq. A6 gives: $B i=0.087$, which is less than 0.1, fulfilling the criterion of the lumped capacitance model uniform temperature for our molten salt.

\section{Supporting Information References:}

(1) Wingert, M. C.; Zhao, A. Z.; Kodera, Y.; Obrey, S. J.; Garay, J. E. Frequency-Domain Hot-Wire Sensor and 3D Model for Thermal Conductivity Measurements of Reactive and Corrosive Materials at High Temperatures. Rev. Sci. Instrum. 2020, 91, 054904.

(2) Bergman, T.L., Lavine, S.A., Incropera, F.P. and Dewitt, D. . Fundamentals of Heat and Mass Transfer. 7th Edition; John Wiley \& Sons: Hoboken, 2011. 